\title{
Membrane Topology of Yeast Alkaline Ceramidase YPC1
}

\author{
Nagaraju RAMACHANDRA* and Andreas CONZELMANN* ${ }^{1}$ \\ *Department of Biology, University of Fribourg, Switzerland \\ ${ }^{1}$ To whom correspondence should be addressed: Department of Biology, Chemin du Musée \\ 10, CH-1700 Fribourg, Switzerland. Tel. ++41 26300 8630; Fax ++41 26300 9735; E-mail: \\ andreas.conzelmann@unifr.ch
}

Condensed title: Topology of yeast alkaline ceramidase YPC1

Key words: YPC1, ceramidase, topology, cysteine accessibility, CREST superfamily

\begin{abstract}
Abbreviations: DHS, dihydrosphingosine; DTR, dual topology reporter; DTT, dithiothreitol; FOA, 5'-fluoroorotic acid; IPC, inositolphosphorylceramide; LCB, long chain base; MIPC, mannosyl-IPC; $\mathrm{M}(\mathrm{IP})_{2} \mathrm{C}$, inositolphosphoryl-MIPC; NEM, N-ethylmaleimide; PEG-mal, PEG5000-maleimide; PHS, phytosphingosine; RT, room temperature; SCAM ${ }^{\mathrm{TM}}$, substituted cysteine accessibility method for TM determination; TM, transmembrane helix; TNBS, trinitrobenzene sulfonic acid; UBI-mal, ubiquitin-maleimide; VLCFA, very long chain fatty acid; wt, wild type.
\end{abstract}

\section{Synopsis}

Ypc1p and Ydc1p are alkaline ceramide hydrolases, which reside in the ER. Ypc1p can catalyze the reverse reaction, i.e. the condensation of free fatty acids with phytosphingosine or dihydrosphingosine and overexpression of YPC1 or YDC1 can provide enough ceramide synthesis as to rescue the viability of cells lacking the normal acyl-CoA-dependent ceramide synthases. To better understand the coexistence of acyl-CoA dependent ceramide synthases and ceramidases in the ER we investigated the membrane topology of Ypc1p by probing cysteine accessibility of natural and substituted cysteines with membrane non-permeating mass-tagged probes. The N- and C-terminal ends of Ypc1p are oriented towards the lumen and cytosol, respectively. Two of the 5 natural cysteines, Cys27 and Cys219, are essential for enzymatic activity and form a disulfide bridge. The data allow inferring that all amino acids of Ypc1p that are conserved in the pfam PF05875 ceramidase motif and the CREST superfamily are located in or near the ER lumen. Microsomal assays using a lysine-specific reagent show that the reverse ceramidase activity can only be blocked when the reagent has access to Ypc1p from the lumenal side. Overall the data suggest that the active site of Ypc1p resides at the lumenal side of the ER membrane.

\section{Introduction}

The mature sphingolipids of Saccharomyces cerevisiae, namely the inositolphosphorylceramides (IPCs), mannosyl-IPCs (MIPCs) and inositolphosphoryl-MIPCs (M(IP) ${ }_{2} \mathrm{Cs}$ ) are major components of the yeast plasma membrane. Moreover, as in higher eukaryotes, the metabolic intermediates of sphingolipid metabolism have been advocated as signaling molecules in many cell biological phenomena and responses (for review see [1]).

Ceramide is a central metabolic intermediate (Fig. 1), which can result from breakdown of IPCs by Isc1p [2] or can be synthesized from acyl-CoA and long chain bases (LCBs) by ceramide synthases consisting of Lag1p or Lac1p in complex with Lip1p [3-7](Fig. 1). Yeast Lag1p and Lac1p are functionally redundant but concomitant deletion of LAG1 and LAC1 causes a significant growth defect in the genetic background of W303 cells and is lethal in the YPK9 background [3, 4, 8]. Insertion of glycosylation sites and factor Xa protease sites into Lag1p and Lac1p, the catalytically active subunits of the yeast ceramide synthase, generated a topological model comprising eight putative membrane-spanning domains, whereby the Lag motif, 
potentially containing the active site, has most conserved residues embedded inside the membrane [9]. The two substrates of Lag1p and Lac1p are a very long chain fatty acid (VLCFA) coupled to Coenzyme A (CoA) and a LCB, either phytosphingosine (PHS) or dihydrosphingosine (DHS) [10]. VLCFA-CoA is generated at the cytosolic surface of the ER membrane [11], whereas endogenous and exogenous LCBs are generated at the cytosolic and lumenal surface of the ER membrane, respectively [9]. Based on these data it has been suggested that LCBs could access the ceramide synthase active site from both sides of the membrane, whereas VLCFA-CoAs access it from the cytosolic side. However, although likely, it is not yet proven that the resulting ceramide is released into the cytoplasmic leaflet of the ER membrane. Natural and spin-labeled ceramides could rapidly flip-flop in liquid ordered giant unilamellar vesicles [12, 13], whereby the relevance of data obtained with these highly artificial systems to natural membranes remains to be demonstrated. In particular, the flip rate of C26:0-containing ceramides, typical of yeast and presumed to be concentrated in rafts, i.e. in areas of the ER membrane where lipids are present in a liquid ordered state, has not been measured so far. Ypc1p and Ydc1p are two highly homologous alkaline ceramidases showing 54\% identity over their entire length [14, 15](Fig. 1). They are predicted to have 7 membrane helices (TMs)(Fig. S1). Overexpression of YPC1 or YDC1 suppresses the lethality of the lag1 $\triangle$ lac1 $\triangle$ double mutation $[5,16]$ by allowing ceramide biosynthesis through the reverse ceramidase reaction, i.e. the condensation of fatty acids with PHS or DHS $[14,15]$. Indeed, the reverse reaction catalyzed by Ypc1p can easily be demonstrated in vitro when microsomes or the purified enzyme are incubated with radiolabeled fatty acids and PHS or DHS [14, 15].

The simultaneous presence of acyl-CoA-dependent ceramide synthases and alkaline ceramidases in the ER seems to potentially create a futile circle. Indeed, in wild type (wt) cells, the overexpression of Ypc1p and Ydc1p proteins was shown to significantly increase the levels of free LCBs and LCB-phosphates and to reduce the biosynthetic flow of long chain bases towards mature sphingolipids, whereas deletion of YPC1 caused a significant increase of mature sphingolipids as detected through metabolic labeling with $\left[{ }^{3} \mathrm{H}\right]$ palmitate or $\left[{ }^{3} \mathrm{H}\right]$ serine or through mass spectrometric quantification of LCBs $[14,17]$.

The three alkaline ceramidases ACER1, ACER2 and ACER3 of humans are homologues of YPC1. ACER1 is only present in skin keratinocytes, while ACER3, showing the highest homology with YPC1, is specific for ceramides carrying unsaturated fatty acids, a kind which is not commonly present in human cells [18]. The ubiquitous ACER2 is localized in the Golgi [18, 19] whereas the mammalian acyl-CoA dependent ceramide synthases are localized in the ER [20]. As ACER2 levels are subject to regulation and moderate overexpression of ACER2 increases sphingosine-1-phosphate levels, the potential for a futile circle also exists in mammalian cells [19].

Ypc1p possesses the pfam PF05875 ceramidase motif containing 7 predicted transmembrane helices (TMs). To date this gene family contains 374 members in 211 species ranging from bacteria to man. A recent bioinformatics study revealed some distant similarity between alkaline ceramidases, the PAQR family of receptors, the PER1 GPI remodelase family, and several other gene families with known and unknown functions, all possessing conserved [SxxxH], [D] and $[\mathrm{HxxxH}]$ motifs being located at defined positions at the ends of predicted TMs 2,3 and 7 [17, 21]. The members of this so-called CREST superfamily may all be metal-binding hydrolases [21].

Here we tried to further our understanding of sphingolipid homeostasis by determining the membrane topology of the conserved residues of Ypc1p.

\section{Experimental}

Yeast strains, media, reagents. Strains are listed in supplemental Table SI. Cells were grown at $30{ }^{\circ} \mathrm{C}$ on rich medium (YPD [1\% (w/v) yeast extract, $2 \%(\mathrm{w} / \mathrm{v})$ peptone, $2 \%(\mathrm{w} / \mathrm{v})$ glucose], supplemented with uracil and adenine) or synthetic complete media (YNB-yeast nitrogen base, U.S. BIOLOGICAL) containing $2 \%$ glucose (D) or galactose (Gal) as a carbon source [22]. 
Radiolabeled palmitic acid $\left(\left[{ }^{3} \mathrm{H}\right]\right.$ palmitate, $\left.60 \mathrm{Ci} / \mathrm{mmol}\right)$ was obtained from ANAWA trading SA, phytosphingosine (PHS) from AVANTI POLAR, $N$-ethylmaleimide (NEM) from FLUKA. Vivaspin 2 ultrafiltration devices having a $10 \mathrm{kDa}$ molecular weight cut off were from MILLIPORE. All other reagents used were from suppliers described before [23]. Bioinformatics tools. Conserved amino acids and motifs in Ypc1p were obtained from http://pfam.sanger.ac.uk and http://www.ncbi.nlm.nih.gov/Structure/cdd/cdd.shtml. http://blast.ncbi.nlm.nih.gov/Blast.cgi was used for protein Blast searches. Alignments were done using ClustalW at http://www.ebi.ac.uk/. Topology predictions were obtained from TOPCONS http://topcons.cbr.su.se/. Calculations of $\Delta \mathrm{G}_{\mathrm{mi}}$ values for TMs were obtained from the $\Delta \mathrm{G}$ predictor at http://dgpred.cbr.su.se/ (full protein scan with length correction turned off, allowing TMs from 15 to 30 amino acids in length).

Construction of strains and plasmids. Plasmids and PCR primers are listed in supplemental Tables SII and SIII. The YPK9 lag $\Delta$ lac1 $\Delta$ strain rescued by overexpression of YPC1 (2 $\triangle$.YPC1) was constructed by amplifying the open reading frame of YPC1 by PCR using genomic DNA as a template and primers 872 and 873 (Table SIII). The PCR product was inserted into pGREG533 by homologous recombination [24] yielding plasmid pBF842 having YPC1 behind the GAL1 promoter, N-terminally tagged by a 7xHA tag. This plasmid was transfected into $2 \Delta$.LAC1 cells and the plasmid pBM150-LAC1 was shuffled out by plating cells on medium containing 5'-fluoroorotic acid (FOA). The resulting strain grows on YNBGal. PCR analysis confirmed that $L A C 1$ was absent, indicating that the plasmid pBM150-LAC1 had been shuffled out successfully. In pBF842 the sequence linking the Nterminal 7xHA tag and the YPC1 ORF contains two cysteines, which in some SCAM ${ }^{\mathrm{TM}}$ experiments served as a lumenal control, but also complicated the interpretation of results. Therefore we replaced the 7xHA tag and this linker sequence in the empty pGREG533 by a cysteine-free $\mathrm{His}_{6}-\mathrm{V} 5$ tag. For this, the GAL1 promoter of pGREG533 was amplified with primers 1493 and 1496 resulting in a fragment containing, between a Sal1 and EcoR1 site, the GAL1 promoter followed by a cysteine free $\mathrm{His}_{6}-\mathrm{V} 5$ epitope. This fragment was used to replace the Sal1-EcoR1 fragment of the original pGREG533 vector that contains the GAL1 promoter, the linker and the 7xHA tag. This replacement yielded the (empty) pNAG3 plasmid containing the GAL1 promoter directly followed by the $\mathrm{His}_{6}$-V5 tag. Some YPC1 alleles in pGREG533 were transferred into pNAG3 by cutting with EcoR1-Xho1 and ligating the fragment into similarly digested pNAG3. Mutations of Ypc1p natural cysteines to Ala or Gly and replacement of natural amino acids by cysteine were done using the QuikChange sitedirected mutagenesis kit (STRATAGENE). All mutations and constructs were verified by sequencing at MICROSYNTH, Balgach, Switzerland.

Preparation of microsomes. Microsomes used for protease accessibility, SCAM ${ }^{\mathrm{TM}}$ assays and TNBS derivatization were generated from cells cultured in galactose at $30^{\circ} \mathrm{C}$ overnight to an $A_{600}$ of 3 using needle disruption of spheroplasts as described before [23]. At the end, the microsomes were resuspended in small volumes of buffers $\mathrm{A}, \mathrm{B}$ or $\mathrm{C}$, depending on the experiment: For protease accessibility assays in buffer A (0.2 M sorbitol, $3 \mathrm{mM}$ EDTA, $5 \mathrm{mM}$ $\mathrm{MgCl}_{2}, 0.1 \mathrm{M}$ potassium phosphate, $\mathrm{pH}$ 7.4), for SCAM ${ }^{\mathrm{TM}}$ in buffer B (0.2 M sorbitol, $5 \mathrm{mM}$ $\mathrm{MgCl}_{2}, 0.1 \mathrm{M}$ sodium phosphate, $\mathrm{pH}$ 7.2). For lysine derivatization using TNBS, the microsomes were resuspended in buffer C ( $0.2 \mathrm{M}$ sorbitol, $3 \mathrm{mM}$ EDTA, $5 \mathrm{mM} \mathrm{MgCl}, 0.1 \mathrm{M}$ sodium bicarbonate, $\mathrm{pH}$ 8.5). Protein concentrations were determined using the Bradford reagent.

Protease protection experiments. The $2 \Delta$.YPC1 cells were grown in YNBGal to stationary phase and microsomes were prepared. Aliquots (100 $\mu \mathrm{g}$ protein/sample) were treated with proteinase $\mathrm{K}$ in buffer $\mathrm{A}$ for $30 \mathrm{~min}$ at RT. Reactions were stopped by addition of $1 \mathrm{x}$ Roche complete EDTA-free protease inhibitor cocktail and Pefabloc, EGTA and PMSF to final concentrations of 20, 5 and $20 \mathrm{mM}$, respectively. After 10 min proteins were precipitated by TCA and processed for Western blotting.

Gel electrophoresis and Western blotting. Samples were incubated for $60 \mathrm{~min}$ at RT in reducing Laemmli sample buffer and separated by SDS-PAGE. Proteins were transferred onto a PVDF membrane using as transfer buffer 25 mM Tris, $192 \mathrm{mM}$ glycine, pH 8.3, 10\% $\mathrm{MeOH}$. 
Cysteine accessibility (SCAM ${ }^{T M}$ ) assays. Plasmid born, cysteine-mutated, epitope-tagged alleles of Ypc1p were expressed in yy $\Delta \Delta$ strain (FBY1224) or yy $\Delta \Delta$ containing FLAG-Gpi8p (FBY8224), Gpi8p being a better lumenal control than Kar2p [25]. Microsomes were analyzed using ubiquitin-EMCS (UBI-mal) and PEG-mal in presence or absence of detergents as recently described [26]. After incubation in presence of UBI-mal $(0.1 \mathrm{mM})$ or PEG-mal $(0.5 \mathrm{mM})$ for $30 \mathrm{~min}$ on ice water, $40 \mathrm{mM}$ DTT was added. After $10 \mathrm{~min}$ on icewater the derivatization of target proteins was visualized by SDS-PAGE and Western blotting using anti-V5, anti-FLAG and anti-Kar2p antibodies for detection of, Ypc1p, Gpi8p and Kar2p, respectively. Alternatively, we performed SCAM ${ }^{\mathrm{TM}}$ assays using NEM adapting protocols described previously [27, 28]. For this, microsomes (100 $\mu \mathrm{g}$ of protein) were incubated with NEM for 30 min on ice-water unless indicated otherwise. Thereafter, residual NEM was removed by washing the membranes twice in buffer B by sedimentation. Microsomes were then incubated on ice water with $1 \mathrm{mM}$ PEG-mal, except for the negative controls or when indicated otherwise. After 10 min SDS (0.5\% final) or dodecylmaltoside (DDM) were added, and the samples were further incubated with PEG-mal for another 25 min, at RT in the case of samples containing SDS, at $0^{\circ} \mathrm{C}$ for the others. The samples were then quenched with DTT and processed for Western blotting as described above.

Microsome preparation for reverse ceramidase assay. Microsomes were generated roughly as described previously [14]. In short, cells were grown overnight at $30^{\circ} \mathrm{C}$ in galactose to an $A_{600}$ of 2. Microsomes were prepared by glass bead disruption of cells in buffer D (25mM Tris$\mathrm{HCl}$, pH 7.4, $5 \mathrm{mM} \mathrm{CaCl}_{2}$ ) containing 1mM PMSF and 1x Roche complete protease inhibitor cocktail. After removal of cell wall debris by low speed centrifugation, microsomes were sedimented at 16 '000 $\mathrm{x} g$ for $30 \mathrm{~min}$ and resuspended in buffer E ( $25 \mathrm{mM}$ Tris-HCl, $\mathrm{pH}$ 8.0, 5 $\mathrm{mM} \mathrm{CaCl}_{2}$ ). Solubilization was done with $0.5 \% \mathrm{v} / \mathrm{v}$ Triton X-100 on ice for $60 \mathrm{~min}$, the lysates were centrifuged at $16,000 \mathrm{x} g$ for $30 \mathrm{~min}$ at $4^{\circ} \mathrm{C}$ and the supernatant was utilized for the assays.

Reverse ceramidase assay. For in vitro assessment of the enzymatic activity of ypc1 alleles, we chose to measure reverse ceramidase activity, which can be assayed using commercially available and relatively water soluble substrates. We used the method described previously [14] with minor modifications: Assays using microsomes from 2 $\Delta$.YPC1 contained $5 \mathrm{nmol}$ PHS, $2 \mu \mathrm{Ci}$ of $\left[{ }^{3} \mathrm{H}\right]$ palmitic acid $(0.3 \mathrm{nmol}$ total). Assays of microsomes from yy $\Delta \Delta$ having different Ypc1p alleles contained $10 \mathrm{nmol}$ PHS and $10 \mu \mathrm{Ci}$ of $\left[{ }^{3} \mathrm{H}\right]$ palmitic acid $(0.23 \mathrm{nmol}$ total). These ingredients were dried under vacuum in a rotary evaporator and dried lipids were dissolved in buffer $\mathrm{E}$ by water bath sonication. Reactions were started by the addition of microsomes or microsomal detergent extract. 100 and $10 \mu \mathrm{g}$ of microsomal protein from $2 \Delta$.YPC1 and yy $\Delta \Delta$.YPC1 cells, respectively, were used for an assay based on preliminary experiments (supplemental Fig. S2). Volumes were complemented to $200 \mu \mathrm{l}$, and samples were incubated at $30^{\circ} \mathrm{C}$ for $120 \mathrm{~min}$ at $300 \mathrm{rpm}$ on a tube shaker. The reactions were terminated by adding $780 \mu \mathrm{l}$ of chloroform/methanol [2:1 (v/v)]. Lipids were extracted, desalted and mild base treated as recently described [29]. Aliquots of each sample were applied to Silica gel 60 plates, which were developed by ascending TLC in $\mathrm{CHCl}_{3} / \mathrm{CH}_{3} \mathrm{OH} / 25 \%$ ammonium hydroxide, 9:2:0.5. Radioactivity was visualized and quantified by two-dimensional radioscanning using a Berthold $\circledast$ radioscanner. Radioactivity contained in $\mathrm{N}-\left[{ }^{3} \mathrm{H}\right]$ palmitoyl-PHS was expressed as a percentage of total radioactivity in the TLC lane. Means and standard deviations of all values obtained through 2 - 4 independent experiments done in duplicate were calculated.

DTR analysis. Dual topology reporters (DTR) were added to the C-terminus of C-terminally truncated versions of YPC1 using homologous recombination and constructs were analyzed as described [26, 30].

Lysine derivatization with TNBS. Microsomes from 2 $\Delta$.YPC1 (FBY8183) (100 $\mu \mathrm{g} / \mathrm{sample})$ in buffer $\mathrm{C}$ were left on ice-water for $15 \mathrm{~min}$ and centrifuged at $16^{\prime} 000 \times g$ for $30 \mathrm{~min}$ at $4^{\circ} \mathrm{C}$. After a second wash, the microsomes were again resuspended in buffer $\mathrm{C}$ and incubated with or without Triton X-100 (0.1\% v/v) for 15 min on ice-water. After addition of TNBS $(0.2 \%$, $\mathrm{v} / \mathrm{v}$ ) or buffer, tubes were further incubated on ice-water for $30 \mathrm{~min}$. Thereafter TNBS was quenched by adding $50 \mathrm{mM}$ Tris pH 8.8, $50 \mathrm{mM}$ imidazole and $100 \mathrm{mM}$ lysine. After $40 \mathrm{~min}$, 
TNBS treated membranes or lysates were either used for a reverse ceramidase assay as described above or treated with proteinase $\mathrm{K}(\mathrm{PK})$ for $30 \mathrm{~min}$ at $0^{\circ} \mathrm{C}$. Thereafter, proteinase $\mathrm{K}$ was blocked with inhibitors and TCA precipitation and proteins processed for Western blotting as described above.

\section{Results}

Cysteines at positions 27 and 219 are required for reverse ceramidase activity SCAM $^{\mathrm{TM}}$ designates a variation of the SCAM (substituted cysteine accessibility method) adapted for determining transmembrane segment orientation of polytopic membrane proteins [31-34]. When polytopic membrane proteins of microsomes are studied by $\mathrm{SCAM}^{\mathrm{TM}}$, the reaction of cysteines with an non-permeating reagent indicate that they are in contact with water and located at the cytosolic side. To reduce ambiguities, one ideally analyzes alleles, which have a single or only few cysteine residues and still preserve good functionality. Ypc1p contains 5 cysteines. Removing the 35 and 74 amino acids from the N- or C-terminal end of Ypc1p, thereby removing Cys27 or Cys271, respectively, yielded non-functional ypc1 alleles that could not rescue YPK9 lag1 $\operatorname{lac} 1 \Delta$ ypc1 $1 \Delta d c 1 \Delta(4 \Delta)$ cells (Fig. 2A). All of the 42 members of the pfam PF05875 ceramidase family conserve Cys219 of Ypc1p. Indeed, replacing Cys219 as well as replacing Cys27 of Ypc1p with Ala created non-functional alleles (Fig. 2B). On the other hand, replacement of the three remaining cysteines (Cys107, Cys115 and Cys271) created ypc1 alleles that still could rescue YPK9 $4 \Delta$ cells. Microsomal reverse ceramidase activity assays using such alleles confirmed that only Cys27 and Cys219 are required for enzymatic activity (Fig. 2C). Treating microsomes with the Cys-specific, membrane-permeating alkylating agent N-ethylmaleimide (NEM) abolished the reverse ceramidase activity of wt Ypc1p, whereas the activity of an allele retaining only the two essential cysteines C27 and C219 was NEM resistant. This indicates that derivatization of one or several of the three non-essential cysteines interferes with the activity.

\section{The N-terminus of Ypc1p is in the ER lumen}

In the absence of detergent, microsomal HA-Ypc1p was quite resistant to proteinase K (Fig. $3 \mathrm{~A}$ ), although treatment with very high concentrations of proteinase $\mathrm{K}$ led to the appearance of a degradation product of lower molecular weight (not shown). As N-terminal tags often are not folded into the protein core, these results suggested that the N-terminus of this fully functional allele resides in the ER lumen. To confirm this notion, we analyzed by SCAM ${ }^{\mathrm{TM}}$ a cysteine-free HA-Ypc1p version carrying two cysteines in the linker region between the N-terminal 7xHA tag and the Ypc1p sequence. For SCAM ${ }^{\mathrm{TM}}$ analysis we used a novel mass-tagged maleimide derivative consisting of ubiquitin coupled to the lysine/cysteine heterobifunctional crosslinker EMCS, dubbed UBI-mal [26, 34]. The two cysteines in the linker region of HA-Ypc1p were accessible to UBI-mal only in the presence of mild detergent but not in its absence (Fig. 3B, lanes 2 and 3). This result also supports the notion that the N-terminus of Ypc1p is ER lumenal.

\section{Cysteine 271 is the only accessible Cys in wild type Ypc1p}

$\mathrm{SCAM}^{\mathrm{TM}}$ analysis of microsomes containing $\mathrm{His}_{6}-\mathrm{V} 5$-tagged wt Ypc1p showed that amongst its 5 cysteines only one reacts with UBI-mal in the absence of detergent (Fig. 4A, lane 2). This cysteine must be located in a cytosolic loop. In the presence of the mild detergent dodecylmaltoside (DDM), a second cysteine was also slightly derivatized. Similarly, the utilization of PEG5000-maleimide (PEG-mal) as a mass tag showed that no more than one cysteine of wt Ypc1p was readily accessible, whether or not DDM was added (Fig. 4A, lanes 68). Addition of SDS revealed that a maximum of 3 out of the 5 cysteines of Ypc1p could be derivatized (Fig. 4A, lane 5). Kar2p, a soluble Hsp70 of the ER lumen has a single cysteine residue and served as a control. It showed that lumenal cysteines became accessible only in the presence of DDM or SDS, albeit not of digitonin (Fig. 4A, lower panel). As reported before, the single cysteine of Kar2p is poorly accessible in the native protein, but becomes more accessible 
in presence of the denaturing detergent SDS (Fig. 4A, lanes 3, 5, 8)[25, 34]. We also used an alternative approach, which avoids the use of mild detergents as a means to remove the membrane barrier. For this, microsomes (containing the proteins in their native state) are exposed to the membrane-permeating NEM so that cysteines on both sides of the membrane are alkylated. One then removes residual NEM and adds a mass-tagged maleimide derivative (UBImal or PEG-mal) in presence of the denaturating detergent SDS [27, 28]. Those cysteines that are not alkylated by NEM when the protein is in the native state, but which get derivatized by a mass-tagged maleimide after denaturation, are classified as "buried" in the native protein structure. Only those cysteines that, in the native structure, are accessible to both NEM and water will not score as buried, since the alkylation reaction of cysteines by NEM also requires the presence of water. As for wt Ypc1p, this alternative approach again showed that UBI-mal derivatized up to three cysteines in the presence of SDS but only a single cysteine in the absence of detergent (Fig. 4B, lanes 1, 2, 6). Pretreatment with $1 \mathrm{mM}$ NEM readily abolished the reactivity of this latter cysteine (Fig. 4B, lane 3), but not of the two other cysteines (Fig. 4B, lane 4). These two buried cysteines remained inaccessible also to higher concentrations of NEM (Fig. 4B, lanes 5 and 8). Thus, the two SCAM ${ }^{\mathrm{TM}}$ approaches used in Fig. 4A and 4B gave the same result: Of the five cysteines of Ypc1p, one is in a cytosolic loop, two are buried and the last two are derivatized under no circumstance.

As expected, a non-functional, cysteine-free version of Ypc1p was derivatized neither by UBImal nor by PEG-mal, and the presence of detergents also did not uncover any reactive residues (Fig. 4C). A ypc1 allele in which Gly replaced C271 could neither be derivatized in absence of mild detergent (DDM) nor in its presence (Fig. 4D, lanes 1 - 3). Denaturation with SDS however rendered up to two cysteines accessible (Fig. 4D, lane 4). The result argued that the one cytosolic cysteine of wt Ypc1p, which is accessible to UBI-mal or PEG-mal in the absence of any detergent (Fig. 4A, lanes 1, 2, 7) is Cys271.

\section{Cysteines 27 and 219 form a disulfide link}

Two cysteines remained inaccessible both in wt and the C271G allele even in presence of SDS (Fig. 2A, B and D) and we guessed that this maybe due to the formation of a disulfide bond between those two cysteines that are essential for function, Cys27 and Cys219. Indeed, a ypc127-219 allele retaining only these two cysteines (CGGCG) could not be derivatized with PEGmal even after denaturation with SDS (Fig. 4E, lanes 1, 3, 5, 7). On the other hand, after prior reduction with dithiothreitol (DDT) these two cysteines became accessible (Fig. 4E, lanes 2, 4, 6, 8). This strongly suggests that a disulfide bond links these two cysteines in Ypc1p-27-219 and also in wt Ypc1p and supports the notion that the N-terminus of Ypc1p is located in the ER lumen. Generally speaking, Cys 27 and Cys219 both are absolutely conserved in the 42 founding members of the pfam05875 motif (Fig. 9), suggesting that the disulfide bridge is conserved throughout the ceramidase family.

\section{Topology of loops L1-2 and L2-3 of Ypc1p}

For further topological studies we decided to substitute individual residues in predicted cytosolic or lumenal loops (Fig. S1) with a cysteine in the Ypc1p allele retaining only the two disulfide linked cysteines, Cys27 and C219 (ypc1-27-219). For this we preferentially chose nonconserved residues in regions, for which the secondary structure was predicted to be random coil. The functionality of the constructs was tested by introducing them into $4 \Delta$.LAG1, a lag1 $\Delta$ lac1 $1 \Delta$ yc $1 \Delta$ ydc1 $1 \Delta$ strain, which dies when it looses the LAG1-containing URA3 plasmid pBM150-LAG1. As shown in supplemental Fig. S3, all substitutions created functional alleles, which rescued growth of $4 \Delta$ cells on FOA with the exception of the K67C and G235C alleles. (FOA kills cells retaining the covering pBM150-LAG1 plasmid). Moreover, the ypc1-27-91-219 allele carrying the K91C mutation provided only limited growth to $4 \Delta$ cells. It should be noted that the rescue of $4 \Delta$ cells requires massive overexpression of YPC1 $[5,16]$, so that any reduction of the catalytic activity of an allele is expected to result in reduced growth of $4 \Delta$ cells. To determine the topology of loops between TM1 and TM2 (loop L1- 2) we analyzed microsomes from yy $\Delta \Delta$ cells harboring a ypc1-27-65-219 or ypc1-27-67-219 allele using both approaches, opening membranes with mild detergent as in Fig. 4A or pretreating them with 
NEM as in Fig. 4B. Cysteines in positions 65 and 67 are not directly accessible when microsomes are incubated with PEG-mal but become accessible in presence of mild detergent (Fig. 5B, lane 2 vs. 7; Fig. 5C, lane 2 vs. 12). Also, NEM is able to prevent their subsequent derivatization with PEG-mal, arguing that they are not buried but exposed at the ER lumenal side of the membrane (Fig. 5B, lane 4; Fig. 5C, lanes 3 - 10). As a control in most of the following SCAM ${ }^{\mathrm{TM}}$ experiments we used a FLAG-tagged Gpi8p allele. Gpi8p is the core protease of the 5-subunit GPI transamidase complex. It is a $50 \mathrm{kDa}$ type I membrane glycoprotein having its N-terminus and all of its 4 Cys on the lumenal side of the ER membrane with only 14 amino acids being displayed at the cytosolic side of the membrane [35]. Up to 4 cysteines were accessible to PEG-mal, when Gpi8p was denatured with SDS (at $25^{\circ} \mathrm{C}$ ), up to 3 when the membrane was permeabilized by DDM at $0^{\circ} \mathrm{C}$ (Fig. $5 \mathrm{~B}$ and $\mathrm{E}$, lanes 6 and 7). Pretreatment with NEM confirmed that only one cysteine of Gpi8p is buried inside the protein and cannot be reached by $10 \mathrm{mM} \mathrm{NEM}$ at $0^{\circ} \mathrm{C}$, although at a higher temperature $\left(25^{\circ} \mathrm{C}\right)$ even this cysteine becomes accessible to NEM (Fig. 5B and E, lanes 4 and 5). As these controls indicate that NEM efficiently penetrated into the ER lumen, the data obtained in Fig. 5B and C argue that L1-2 is lumenal. The same pattern, characteristic for lumenally exposed cysteines, was also observed for two cysteines introduced at positions 91 and 93 in loop L2-3, a very short loop comprising only amino acids 91 - 94 according to the current TOPCONS global prediction (Fig. 5A, E, F). This prediction also places T89 to the end of TM2 and indeed, we found the T89C cysteine of ypc1-27-89-219 allele to be buried, although it became partially accessible to $\mathrm{NEM}$ at $25^{\circ} \mathrm{C}$ (Fig. 5D, lanes 4 and 5). Overall, the data clearly indicate that loops L1-2 and L23 are lumenal.

\section{Topology of loops L3-4, L4-5, L5-6 and L6-7 of Ypc1p}

Using the same approach as described above we found that cysteines replacing N123 and G124 both were immediately accessible to PEG-mal, in the absence of any detergent (Fig. 6B and C). This results shows that L3-4 is cytosolic (Fig. 6A).

Loop L4-5 is very short and comprises only amino acids 157 to 159 according to TOPCONS (Fig. 6A). It was probed by introducing cysteines at positions 157 and 158. These cysteines were not accessible in the absence of detergent, but accessible to NEM, arguing that they are lumenally exposed (Fig. 6D and E).

Loop L5-6 comprises amino acids 181-195 according to TOPCONS and was probed by substituting cysteines at positions 181,182, 184 and 185, all of which seemed to be located at the lumenal side of the ER (Fig. 7A-E). S197 residing at the beginning of the following TM6 proved to be completely buried (Fig. 7F). Overall, the data indicate a lumenal orientation of L56.

Loop L6-7 comprises, according to TOPCONS, amino acids 217-245. The ypc1-27-219-235 allele is non-functional but SCAM ${ }^{\mathrm{TM}}$ indicates a lumenal position of 235 (Fig. 7G). The ypc127-219-236 allele was classified as partially buried, since part of the protein could still be derivatized with PEG-mal after NEM treatment (Fig. 7H, lanes 4 and 6). Doing the NEM pretreatment at 25 instead of $0^{\circ} \mathrm{C}$, or raising the NEM concentration to $20 \mathrm{mM}$ did not increase the accessibility of Cys236 (Fig. 7H, lanes 4-6, 8, 9). Another way to look at the data is to say that the PEG-mal derivatization of Cys 236 was substantially reduced by NEM pretreatment , indicating that it is partially accessible to NEM (Fig. 7H, lanes 2, 4-6). Similarly Cys 236 was also partially accessible to PEG-mal in presence of DDM (Fig. 7H, lane 7), whereas it was not accessible to PEG-mal in the absence of detergent (Fig. 7H, lane 2). The result is compatible with the idea that there are two forms of Ypc1p, one in which Cys 236 is accessible from the lumenal side of the ER, another where this cysteine is buried, e.g. because another protein interacts with Ypc1p. The lumenal location of L6-7 (Fig. 7A) is supported also by a dual topology reporter (DTR) inserted after P233 showing that the Ypc1p-P233-DTR construct is glycosylated and does not support growth of his $4 \Delta$ cells on histidinol (Fig. 7I). The DTR approach has been shown to be able to yield correct topology prediction only, when the DTR is inserted at a certain distance downstream of a TM having a negative $\Delta \mathrm{G}_{\mathrm{mi}}$ and of sufficient length to span the membrane $[34,36]$. These conditions are met for DTR cassettes truncating Ypc1p after TM6. The existence of a disulfide bond between C27 and C219 also supports a 
lumenal orientation of L6-7 is (Fig. 4E).

\section{The C-terminal end of Ypc1p is cytosolic}

The TOPCONS global prediction places amino acids 267- 316 into the cytosol. Indeed, a global study to locate the C-terminal ends of yeast membrane proteins using DTR has mapped the Cterminal end of Ypc1p to the cytosol [37]. This conclusion is fully supported also by the results obtained with the Ypc1p-P292-DTR construct shown in Fig. 7I. It is further supported by SCAM $^{\mathrm{TM}}$ data showing a cytosolic location of the natural Cys271 (Fig. 4), but also the SCAM ${ }^{\mathrm{TM}}$ data shown in Fig. S4. When generating the ypc1-27-184-219 allele the PCR mutagenesis wrongly introduced an additional mutation, S293C. This ypc1-27-184-219-293 allele contains one cysteine, which reacts with PEG-mal in the absence of detergent (Fig. S4A, lane 2) whereas the corrected ypc127-184-219 does not react in this condition (Fig. 7D, lane 2). Thus, Cys293 must be cytosolic. Similarly, probing an allele having an S297C mutation showed that position 297 is accessible from the cytosolic side of the membrane (Fig. S4B). Overall, all data argue that the C-terminus of Ypc1p is cytosolic, allowing its C-terminal KKEK motif to act as an ER retrieval signal.

\section{Lysine derivatization in the absence of detergent does not interfere with Ypc1p activity} Trinitrobenzene sulfonic acid (TNBS) is a lysine-reactive reagent, which does not penetrate the membrane. When TNBS was added to intact microsomes, the reverse ceramidase activity remained unchanged (Fig. 8A and B, lanes 1 and 3). The addition of TNBS to an assay containing Triton X-100 however caused a drastic reduction of Ypc1p activity (Fig. 8A and $\mathrm{B}$, lanes 2 and 4). When, before being added to microsomes, TNBS was quenched with a mix of lysine, imidazol and Tris, it did not inhibit reverse ceramidase activity (Fig. 8A, lanes 3, 4, $6,7)$. This activity did not decrease even when microsomes were treated with TNBS for up to two hours (not shown). TNBS-derivatized proteins were detected by Western blotting using anti-DNP antibody (Fig. S5A). To localize the TNBS-derivatization on microsomal proteins, TNBS-treated microsomes were treated with proteinase $\mathrm{K}$. When derivatization was performed in presence of Triton X-100, all proteins were almost completely digested by subsequent addition of proteinase K (Fig. S5A, B, C, lanes 3, 6 - 7). Even when TNBS derivatization was done in the absence of detergent, a large part of the TNBS-derivatized proteins were destroyed by proteinase K (Fig. S5A, lanes 2, 4 - 5), whereas $74 \%$ of the ER lumenal Kar2p and $80 \%$ of the lumenally tagged HA-Ypc1p were preserved (Fig. S5B, C). This indicates that TNBS does not efficiently penetrate the ER membrane. The resistance of Kar2p and HA-Ypc1p to protease added from the cytosolic side is not due to the protection of lysines by TNBS, since these proteins were even more resistant when TNBS treatment was omitted (Fig. S5A - C, lanes 4, 5, 8 - 11). Incidentally, the results documented again that Ypc1p is highly resistant to protease added from the cytosolic side of microsomes, whereas it is readily degraded when detergent is present (Figs. 3A, 9E). Ypc1p contains 16 lysine residues, 9 of which are in cytosolic loops, while the other 7 are in lumenal loops, according to our model (Fig. 9). The data argue that the derivatization of cytosolic lysines in the absence of detergent does not interfere with reverse ceramidase activity, whereas derivatization of some lysine in the presence of detergent does, although no single lysine of Ypc1p is conserved.

\section{Discussion}

Ceramides are appearing as intermediates in both biosynthetic and catabolic pathways insuring subcellular and global sphingolipid and lipid homeostasis of eukaryotic cells. In yeast, ceramides are generated either through the acyl-CoA dependent ceramide synthases Lag1p and Lac1p or through degradation of IPCs. Isc1p most certainly generates ceramides from IPCs in the cytosolic leaflet while this is not proven for the ceramide synthases Lag1p and Lac1p. 
Ceramides basically can enter four different pathways (Fig. 1): i) IPC biosynthesis [38], ii) GPI anchor remodeling [39], iii)1-O-acylation generating acylceramides [29], and iv) hydrolysis by ceramidases [14] (Fig. 1). Pathways i-iii have been claimed to occur at the lumenal side of the secretory pathway based on biochemical data and bioinformatic analysis of the location of conserved motifs [27, 29, 40-43]. This report makes it likely that also the degradation via alkaline ceramidases occurs lumenally. Thus, the ER based anabolic pathways leading to integration of ceramides into GPI anchors and acylceramides may compete with ceramidases for ceramides residing in the same leaflet of the bilayer.

A previous study showed that the human alkaline ceramidase ACER2 has the N-terminal end in the lumen, the C-terminus in the cytosol and that the deletion of the $\mathrm{N}$-terminal end, containing residues conserved between yeast and mammalian alkaline ceramidases, abolishes ceramidase activity [19]. The bioinformatic predictions of the membrane topology of ACER2 place the most highly conversed residues of all alkaline ceramidases into the lumen, whereas the $\mathrm{C}$ terminus and three putative cytosolic loops do not contain highly conserved residues. Based on this it was proposed that the presumed active site of ACER2 may be located in the lumen of the Golgi complex [19]. Based on bioinformatics tools, this prediction can be extrapolated to the other two human ceramidases [21](Fig. S6). Overall, almost all residues conserved between YPC1 and its closest fungal as well as metazoan homologues are in or near the ER lumen, according to both, our topology model as well as the global TOPCONS prediction (Fig. S1). Interestingly, part of the residues conserved in the PF05875 ceramidase family are also conserved in the larger CREST superfamily encompassing about 3000 predicted proteins with 7 transmembrane helices (Fig. 9, Fig. S1). Yeast harbors four other proteins belonging to the CREST superfamily, namely the Izh1-Izh4 proteins, three of which have been localized in the ER [44]. Within the CREST superfamily, Izh1-Izh4 proteins are most closely related to the PAQR family, a family that is predicted to have an inverted topology in that all 5 conserved residues are predicted to be cytosolic [21]. The global TOPCONS predictions for Izh1p-Izh4p indeed predict a cytosolic location of conserved residues (Fig. S6), and these predictions have an uncommonly high reliability, since for each Izh protein, all 5 algorithms (corresponding to lines a -e of Fig. S1) indicate the exact same topology (not shown). Interestingly, Izh2p has been shown to be involved in a signaling pathway, which results in the elevation of LCBs, raising the possibility that also this group of proteins acts as ceramidases [17]. If this can be shown biochemically, it would imply that functionally and structurally similar yeast proteins are made with two opposite topologies. While mammalian ceramidase ACER2 has been shown to have the potential of regulating Nglycan modifications and production of sphingosine and sphingosine-1-phosphates in studies where the proteins was overexpressed $[19,45]$, the reasons for the preservation of alkaline ceramidases in fungi so far is not apparent and further efforts are required to understand their physiological roles.

\section{Contributions}

The experimental work was carried out by NR, the planning of experiments and the writing of the manuscript was done jointly by the two authors.

AcknowledgementsWe would like to thank Dr. Martin Pagac and Arlette Bochud for helpful discussions.

\section{Funding}

This work was supported by grants CRSI33_125232 and 31003A_131078 from the Swiss

National Science Foundation (http://www.snf.ch/E/Pages/default.aspx). 


\section{References}

1. Dickson, R. C. (2010) Roles for sphingolipids in Saccharomyces cerevisiae. Adv Exp Med Biol. 688, 217-231

2. Sawai, H., Okamoto, Y., Luberto, C., Mao, C., Bielawska, A., Domae, N. and Hannun, Y. A. (2000) Identification of ISC1 (YER019w) as inositol phosphosphingolipid phospholipase C in Saccharomyces cerevisiae. J Biol Chem. 275, 39793-39798

3. Guillas, I., Kirchman, P. A., Chuard, R., Pfefferli, M., Jiang, J. C., Jazwinski, S. M. and Conzelmann, A. (2001) C26-CoA-dependent ceramide synthesis of Saccharomyces cerevisiae is operated by Lag1p and Lac1p. EMBO J. 20, 2655-2665

4. Jiang, J. C., Kirchman, P. A., Zagulski, M., Hunt, J. and Jazwinski, S. M. (1998) Homologs of the yeast longevity gene LAG1 in Caenorhabditis elegans and human. Genome Res. 8, 1259-1272

5. $\quad$ Schorling, S., Vallee, B., Barz, W. P., Riezman, H. and Oesterhelt, D. (2001) Lag1p and Lac1p are essential for the Acyl-CoA-dependent ceramide synthase reaction in Saccharomyces cerevisae. Mol Biol Cell. 12, 3417-3427

6. Winter, E. and Ponting, C. P. (2002) TRAM, LAG1 and CLN8: members of a novel family of lipid-sensing domains? Trends Biochem Sci. 27, 381-383

7. Vallee, B. and Riezman, H. (2005) Lip1p: a novel subunit of acyl-CoA ceramide synthase. EMBO J. 24, 730-741

8. $\quad$ Barz, W. P. and Walter, P. (1999) Two endoplasmic reticulum (ER) membrane proteins that facilitate ER-to-Golgi transport of glycosylphosphatidylinositol-anchored proteins. Mol Biol Cell. 10, 1043-1059

9. Kageyama-Yahara, N. and Riezman, H. (2006) Transmembrane topology of ceramide synthase in yeast. Biochem J. 398, 585-593

10. Guillas, I., Jiang, J. C., Vionnet, C., Roubaty, C., Uldry, D., Chuard, R., Wang, J., Jazwinski, S. M. and Conzelmann, A. (2003) Human homologues of LAG1 reconstitute Acyl-CoA-dependent ceramide synthesis in yeast. J Biol Chem. 278, 37083-37091

11. Denic, V. and Weissman, J. S. (2007) A molecular caliper mechanism for determining very long-chain fatty acid length. Cell. 130, 663-677

12. Lopez-Montero, I., Rodriguez, N., Cribier, S., Pohl, A., Velez, M. and Devaux, P. F. (2005) Rapid transbilayer movement of ceramides in phospholipid vesicles and in human erythrocytes. J Biol Chem. 280, 25811-25819

13. Pohl, A., Lopez-Montero, I., Rouviere, F., Giusti, F. and Devaux, P. F. (2009) Rapid transmembrane diffusion of ceramide and dihydroceramide spin-labelled analogues in the liquid ordered phase. Mol Membr Biol. 26, 194-204

14. Mao, C., Xu, R., Bielawska, A. and Obeid, L. M. (2000) Cloning of an alkaline ceramidase from Saccharomyces cerevisiae. An enzyme with reverse (CoA-independent) ceramide synthase activity. J Biol Chem. 275, 6876-6884

15. Mao, C., Xu, R., Bielawska, A., Szulc, Z. M. and Obeid, L. M. (2000) Cloning and characterization of a Saccharomyces cerevisiae alkaline ceramidase with specificity for dihydroceramide. J Biol Chem. 275, 31369-31378

16. Jiang, J. C., Kirchman, P. A., Allen, M. and Jazwinski, S. M. (2004) Suppressor analysis points to the subtle role of the LAG1 ceramide synthase gene in determining yeast longevity. Exp Gerontol. 39, 999-1009

17. Villa, N. Y., Kupchak, B. R., Garitaonandia, I., Smith, J. L., Alonso, E., Alford, C., Cowart, L. A., Hannun, Y. A. and Lyons, T. J. (2009) Sphingolipids function as downstream effectors of a fungal PAQR. Mol Pharmacol. 75, 866-875

18. Mao, C. and Obeid, L. M. (2008) Ceramidases: regulators of cellular responses mediated by ceramide, sphingosine, and sphingosine-1-phosphate. Biochim Biophys Acta. 1781, 424-434

19. Sun, W., Jin, J., Xu, R., Hu, W., Szulc, Z. M., Bielawski, J., Obeid, L. M. and Mao, C. (2010) Substrate specificity, membrane topology, and activity regulation of human alkaline ceramidase 2 (ACER2). J Biol Chem. 285, 8995-9007

20. Pewzner-Jung, Y., Ben-Dor, S. and Futerman, A. H. (2006) When do Lasses (longevity 
assurance genes) become CerS (ceramide synthases)?: Insights into the regulation of ceramide synthesis. J Biol Chem. 281, 25001-25005

21. Pei, J., Millay, D. P., Olson, E. N. and Grishin, N. V. (2011) CREST--a large and diverse superfamily of putative transmembrane hydrolases. Biol Direct. 6, 37

22. Sherman, F. (2002) Getting started with yeast. Methods Enzymol. 350, 3-41

23. Pagac, M., Vazquez, H. M., Bochud, A., Roubaty, C., Knopfli, C., Vionnet, C. and Conzelmann, A. (2012) Topology of the microsomal glycerol-3-phosphate acyltransferase Gpt2p/Gat1p of Saccharomyces cerevisiae. Mol Microbiol. 86, 1156-1166

24. Jansen, G., Wu, C., Schade, B., Thomas, D. Y. and Whiteway, M. (2005) Drag\&Drop cloning in yeast. Gene. 344, 43-51

25. Bochud, A., Ramachandra, N. and Conzelmann, A. (2013) Adaptation of low-resolution methods for the study of yeast microsomal polytopic membrane proteins: a methodological review. Biochem. Soc. Trans. 41, in press

26. Pagac, M., de la Mora, H. V., Duperrex, C., Roubaty, C., Vionnet, C. and Conzelmann, A. (2011) Topology of 1-acyl-sn-glycerol-3-phosphate acyltransferases SLC1 and ALE1 and related membrane-bound O-acyltransferases (MBOATs) of Saccharomyces cerevisiae. J Biol Chem. 286, 36438-36447

27. Liu, Q., Siloto, R. M., Snyder, C. L. and Weselake, R. J. (2011) Functional and topological analysis of yeast acyl-CoA:Diacylglycerol acyltransferase 2, an endoplasmic reticulum enzyme essential for triacylglycerol biosynthesis. J Biol Chem. 286, 1311513126

28. Wang, Y., Toei, M. and Forgac, M. (2008) Analysis of the membrane topology of transmembrane segments in the C-terminal hydrophobic domain of the yeast vacuolar ATPase subunit a (Vph1p) by chemical modification. J Biol Chem. 283, 20696-20702

29. Voynova, N. S., Vionnet, C., Ejsing, C. S. and Conzelmann, A. (2012) A novel pathway of ceramide metabolism in Saccharomyces cerevisiae. Biochem J. 447, 103-114

30. Kreft, S. G., Wang, L. and Hochstrasser, M. (2006) Membrane topology of the yeast endoplasmic reticulum-localized ubiquitin ligase Doa10 and comparison with its human ortholog TEB4 (MARCH-VI). J Biol Chem. 281, 4646-4653

31. Falke, J. J., Dernburg, A. F., Sternberg, D. A., Zalkin, N., Milligan, D. L. and Koshland, D. E. J. (1988) Structure of a bacterial sensory receptor. A site-directed sulfhydryl study. J Biol Chem. 263, 14850-14858

32. Karlin, A. and Akabas, M. H. (1998) Substituted-cysteine accessibility method. Methods Enzymol. 293, 123-145

33. Bogdanov, M., Zhang, W., Xie, J. and Dowhan, W. (2005) Transmembrane protein topology mapping by the substituted cysteine accessibility method (SCAM(TM)): application to lipid-specific membrane protein topogenesis. Methods. 36, 148-171

34. Bogdanov, M. and Dowhan, W. (1999) Lipid-assisted protein folding. J Biol Chem. 274, 36827-36830

35. Benghezal, M., Benachour, A., Rusconi, S., Aebi, M. and Conzelmann, A. (1996) Yeast Gpi8p is essential for GPI anchor attachment onto proteins. EMBO J. 15, 6575-6583

36. Cassel, M., Seppala, S. and von Heijne, G. (2008) Confronting fusion protein-based membrane protein topology mapping with reality: the Escherichia coli ClcA H+/Clexchange transporter. J Mol Biol. 381, 860-866

37. Kim, H., Melen, K., Osterberg, M. and von Heijne, G. (2006) A global topology map of the Saccharomyces cerevisiae membrane proteome. Proc Natl Acad Sci U S A. 103, 11142-11147

38. Nagiec, M. M., Nagiec, E. E., Baltisberger, J. A., Wells, G. B., Lester, R. L. and Dickson, R. C. (1997) Sphingolipid synthesis as a target for antifungal drugs. Complementation of the inositol phosphorylceramide synthase defect in a mutant strain of Saccharomyces cerevisiae by the AUR1 gene. J Biol Chem. 272, 9809-9817

39. Fankhauser, C., Homans, S. W., Thomas-Oates, J. E., McConville, M. J., Desponds, C., Conzelmann, A. and Ferguson, M. A. (1993) Structures of glycosylphosphatidylinositol membrane anchors from Saccharomyces cerevisiae. J Biol Chem. 268, 26365-26374

40. Levine, T. P., Wiggins, C. A. and Munro, S. (2000) Inositol phosphorylceramide synthase 
is located in the Golgi apparatus of Saccharomyces cerevisiae. Mol Biol Cell. 11, 22672281

41. Umemura, M., Fujita, M., Yoko-O, T., Fukamizu, A. and Jigami, Y. (2007)

Saccharomyces cerevisiae CWH43 is involved in the remodeling of the lipid moiety of GPI anchors to ceramides. Mol Biol Cell. 18, 4304-4316

42. Ghugtyal, V., Vionnet, C., Roubaty, C. and Conzelmann, A. (2007) CWH43 is required for the introduction of ceramides into GPI anchors in Saccharomyces cerevisiae. Mol Microbiol. 65, 1493-1502

43. Choudhary, V., Jacquier, N. and Schneiter, R. (2011) The topology of the triacylglycerol synthesizing enzyme Lro1 indicates that neutral lipids can be produced within the luminal compartment of the endoplasmatic reticulum: Implications for the biogenesis of lipid droplets. Commun Integr Biol. 4, 781-784

44. Huh, W. K., Falvo, J. V., Gerke, L. C., Carroll, A. S., Howson, R. W., Weissman, J. S. and O'Shea, E. K. (2003) Global analysis of protein localization in budding yeast. Nature. 425, 686-691

45. Xu, R., Jin, J., Hu, W., Sun, W., Bielawski, J., Szulc, Z., Taha, T., Obeid, L. M. and Mao, C. (2006) Golgi alkaline ceramidase regulates cell proliferation and survival by controlling levels of sphingosine and S1P. FASEB J. 20, 1813-1825

46. Bernsel, A., Viklund, H., Hennerdal, A. and Elofsson, A. (2009) TOPCONS: consensus prediction of membrane protein topology. Nucleic Acids Res. 37, W465-8

\section{Figure legends}

Fig. 1. Ceramide biosynthesis and utilization in yeast. A, The various metabolic pathways generating and consuming ceramides are shown. Gene names are in italic. $\mathbf{B}$, TOPCONS is one of the most recent bioinformatic tools for the prediction of TMs [46]. Lines a - e show the topologies predicted for Ypc1p by the 5 TOPCONS algorithms SCAMPI-seq, SCAMPI-msa, PRODIV, PRO and OCTOPUS. The TOPCONS global prediction (line f) takes into account two further algorithms ( $\triangle \mathrm{G}_{\mathrm{mi}}$ and ZPRED) and differs slightly from the topology proposed by the present report (line g). Potential TMs are numbered 1-7 above line a, their theoretical $\Delta \mathrm{G}_{\mathrm{mi}}$ values $(\mathrm{kcal} / \mathrm{mol})$ obtained from the $\Delta \mathrm{G}$ predictor are indicated below line g. Sequences predicted to be lumenal and cytosolic are in blue and red, respectively.

Fig. 2. Cysteines at positions 27 and 219 are required for reverse ceramidase activity. A, the YPK9 4A.LAG1 (FBY2171) strain was transfected with wt YPC1 or ypc1 alleles having Nor C-terminal truncations up to the indicated amino acids. Ten fold dilutions of cells were plated onto YNBGal with or without 5'-fluoroorotic acid (FOA). On FOA, only cells having lost the pBM150-LAG1 (URA3) plasmid can survive. Plates were incubated for 3 days at $30^{\circ} \mathrm{C}$. B, the same strain was transfected with ypc1 alleles in which part of the five natural cysteines were replaced by Ala or Gly. Their exact positions are indicated at the top with positions containing conserved cysteines being in red. Functionality of the alleles was tested as in panel A. C, some alleles tested in panel B were transfected into yy $\Delta \Delta$ strain (FBY1224) and microsomes were used for an in vitro microsomal reverse ceramidase assay. Samples were run out on TLC, radioactivity was detected by radioscanning and $\mathrm{N}-\left[{ }^{3} \mathrm{H}\right]$ palmitoyl-PHS expressed as $\%$ of total counts per lane. Means of two independent assays done in duplicate are shown with standard deviations. D, $100 \mu \mathrm{g}$ of microsomes from yy $\Delta \Delta$ cells harboring the wt CCCCC (FBY8203) or the CGGCG allele (FBY8204) of YPC1 were incubated with $15 \mathrm{mM}$ NEM or ethanol for control during $30 \mathrm{~min}$ on ice-water. Microsomes were sedimented $\left(16^{\prime} 000 \mathrm{~g} \mathrm{x} 30 \mathrm{~min}\right)$ at $4^{\circ} \mathrm{C}$. The pellet was resuspended in $200 \mu \mathrm{l}$ of buffer E containing $20 \mathrm{mM}$ DTT and further incubated for $10 \mathrm{~min}$ on ice-water. Ten $\mu \mathrm{g} / \mathrm{sample}$ were used for Ypc1p reverse activity assays. Data were analyzed as in panel C, means of 3 independent assays done in duplicates were plotted.

Fig. 3. The $\mathbf{N}$ terminus of Ypc1p is in the lumen of the ER. A, microsomes from $2 \Delta$.YPC1 
cells containing HA-Ypc1p with a 7xHA tag at the N-terminus were incubated without or with the indicated amounts of proteinase $\mathrm{K}(\mathrm{PK})$ in the presence or absence of Triton X-100 $(0.5 \%$, $\mathrm{v} / \mathrm{v}$ ) for $30 \mathrm{~min}$ at room temperature (RT) and products were analyzed by Western blotting using anti-HA and anti-Kar2p antibodies. B, microsomes from yy $\Delta \Delta$ cells harboring pBF849 were used. pBF849 carries a cysteine-free (AGGAG) HA-Ypc1p allele with two non-natural cysteines residing in the linker between the 7xHA tag and YPC1. These non-natural cysteines were localized by SCAM ${ }^{\mathrm{TM}}$ incubating samples with UBI-mal, in presence of $0.66 \%$ DDM (D), $0.16 \%$ SDS.

Fig. 4. Cysteines 27 and 219 of Ypc1p are forming an S-S bridge. A, microsomes from yy $\Delta \Delta$ cells transfected with wt $\mathrm{His}_{6}$-V5-YPC1 were treated with UBI-mal or PEG-mal in presence of buffer (-), digitonin (Dt, 0.66\%), DDM (D, 0.66\%) or SDS (S, 0.16\%). In B, the same microsomes were pretreated with 1,5 or $10 \mathrm{mM}$ NEM at $0^{\circ} \mathrm{C}$ and then washed twice before being treated with UBI-mal $(0.1 \mathrm{mM})$ in the presence or absence of SDS (S, 0.5\%). C and $\mathbf{D}$, as panel A but using microsomes containing the cysteine-free $\mathrm{His}_{6}-\mathrm{V} 5-\mathrm{Ypc1p}$ (C) or a $\mathrm{His}_{6}$-V5Ypc1p lacking C271 (D). E, microsomes from yy $\Delta \Delta$ cells having His $_{6}-\mathrm{V} 5-$ Ypc1p-27-219 (pBF85) were solubilized in 0.5\% SDS in the presence and absence of DTT $(25 \mathrm{mM})$ for $60 \mathrm{~min}$ at RT. Upon removal of DTT by ultrafiltration the lysates were treated with $0,1,3$ or $5 \mathrm{mM}$ PEG-mal. The protein extracts were processed as above.

Fig. 5. Topology of loops L1-2 and L2-3. A, TM predictions for the first 130 amino acids of Ypc1p by the TOPCONS global prediction (line f) and the one proposed by the present report (line g) as described in Fig. S1. Positions of amino acid residues replaced by cysteines are marked in green. B-F, microsomes from FBY8228 (B), FBY8229 (C), FBY8263 (D), FBY8230 (E) and FBY8231 (F) were incubated for 30 min with 0 - $20 \mathrm{mM}$ NEM at indicated temperature $\left({ }^{\circ} \mathrm{C}\right)$ and processed for $\mathrm{SCAM}^{\mathrm{TM}}$.

Fig. 6. Topology of loops L3-4 and L4-5. A, TM predictions for amino acids 90-180 of Ypc1p as in Fig. 5A. B-E, microsomes from FBY8233 (B), FBY8234 (C), FBY8264 (D) and FBY8235 (E) cells were incubated for $30 \mathrm{~min}$ with 0 or $10 \mathrm{mM} \mathrm{NEM}$ at $0^{\circ} \mathrm{C}$ and processed for SCAM ${ }^{\mathrm{TM}}$.

Fig. 7. Topology of loops L5-6 and L6-7. A, TM predictions for amino acids 160-270 of Ypc1p as in Fig. 5A. B-H, microsomes from FBY8236 (B), FBY8237 (C), FBY8238 (D), FBY8239 (E), FBY8240 (F), FBY8241 (G) and FBY8242 (H) cells were incubated for 30 min with 0 - 20 $\mathrm{mM}$ NEM at indicated temperatures and processed for SCAM ${ }^{\mathrm{TM}}$. I, DTR constructs containing amino acids 1 to 233 or 292 of Ypc1p followed by the DTR (3xHA-Suc2-His4C cassette) were transfected into his4 $\Delta$ (STY50) cells. Left panel, serial ten fold dilutions of cells were plated on minimal medium containing either histidine or its precursor, histidinol. (His $4 \Delta$ grow on histidinol, if the DTR cassette is on the cytosolic side of the ER membrane, not if it is lumenal.) Right panel, proteins of exponentially growing cells were extracted, incubated with $(+)$ or without (-) endoglycosidase $\mathrm{H}$, separated by SDS-PAGE and processed for Western blotting using anti-HA antibody.

Fig. 8. Ypc1p activity is blocked by TNBS in the presence of detergent. A, microsomes from $2 \Delta$.YPC1 were incubated with or without TNBS $(0.2 \%, \mathrm{v} / \mathrm{v})$ in the presence or absence of Triton $\mathrm{X}-100(0.1 \% \mathrm{v} / \mathrm{v})$ on ice-water. Thereafter TNBS was quenched and ingredients for the reverse ceramidase assay were added. Microsomes in lane 5 were boiled. In lanes 6 and 7 TNBS had been quenched before being added to microsomes. The Berthold radioscans is shown. $\mathbf{B}$, quantification of [ $\left.{ }^{3} \mathrm{H}\right] \mathrm{C} 16$ incorporated into $\mathrm{N}-\left[{ }^{3} \mathrm{H}\right]$ palmitoyl-PHS (Cer) in lanes $1-4$ of panel $\mathrm{A}$, as \% of total counts per lane, and of three independent repeat assays each done in duplicates.

Fig. 9. Topology model for Ypc1p based on this study. Membrane is bluish, the 24 residues considered to be highly conserved in pfam motif PF05875 at Sanger are in red lettering, the 13 residues strictly conserved in the same PF05875 according to NCBI are in red circles, the 5 residues conserved in the CREST family are underlaid in turquoise, the residues which were 
substituted for SCAM are underlaid yellow if the cysteine substitution resulted in a fully functional allele, violet, when substitution compromised the functionality. Non-conserved cysteines are in blue lettering and residues where a DTR was inserted are underlaid green. 


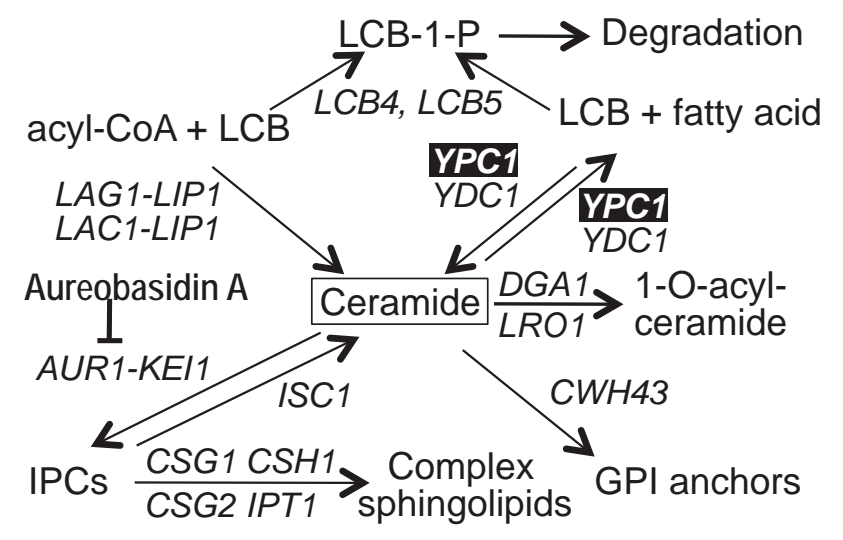

Fig. 1 


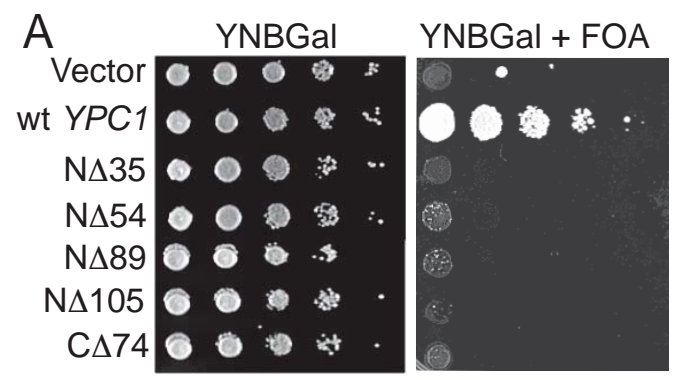

B

wt $Y p c 1 p=\mathbf{C}^{27} \mathrm{C}^{107} \mathrm{C}^{115} \mathrm{C}^{219} \mathrm{C}^{271}=\mathrm{CCCC} C$

\begin{tabular}{|c|c|c|c|c|}
\hline & & YNBGal & & YNBGal + FOA \\
\hline vector & & $00 \%$ & & \\
\hline $\mathbf{C C C C C}$ & 0 & $00 \%$ & 4 & 5 \\
\hline $\mathrm{ACCAC}$ & & 00 & .. & \\
\hline $\mathbf{C G G C G}$ & & $0 \Rightarrow 5$ & $\therefore$ & 001 \\
\hline CGGAG & 0 & $6 \%$ & & \\
\hline CCCAC & 0 & 0. & & • \\
\hline $\mathrm{ACCC}$ & & 0 & & \\
\hline $\begin{array}{l}\mathbf{C} C \mathbf{C G}_{\mathrm{G}} \\
\mathrm{AGGAG}\end{array}$ & & $\begin{array}{l}0 \\
00\end{array}$ & & \\
\hline
\end{tabular}
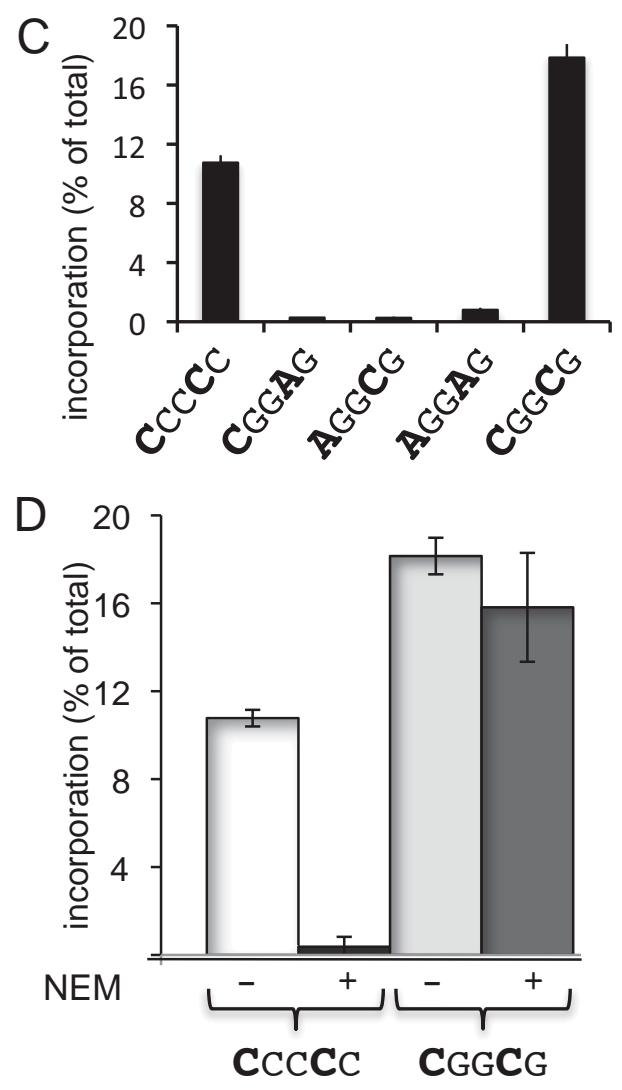

Fig. 2 


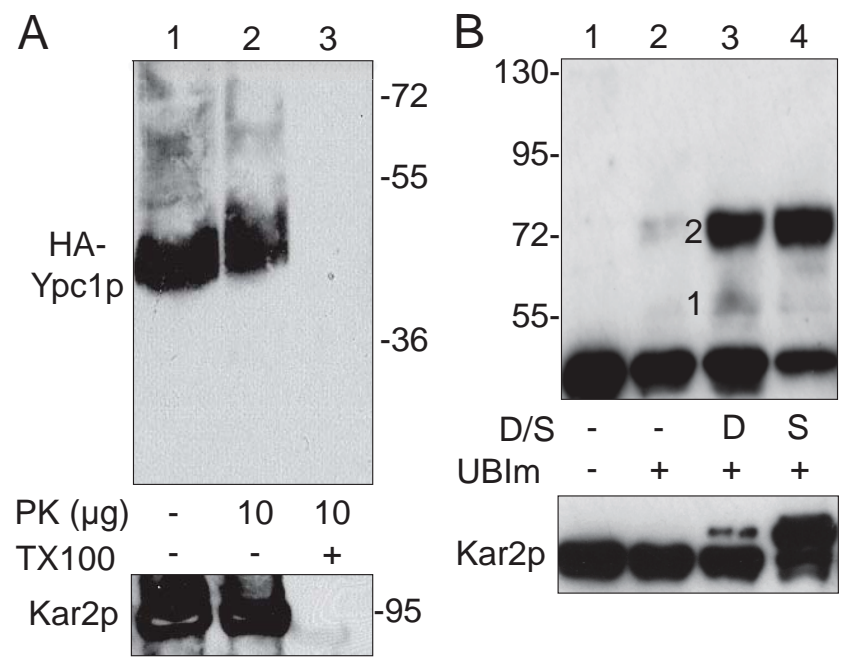

Fig. 3 

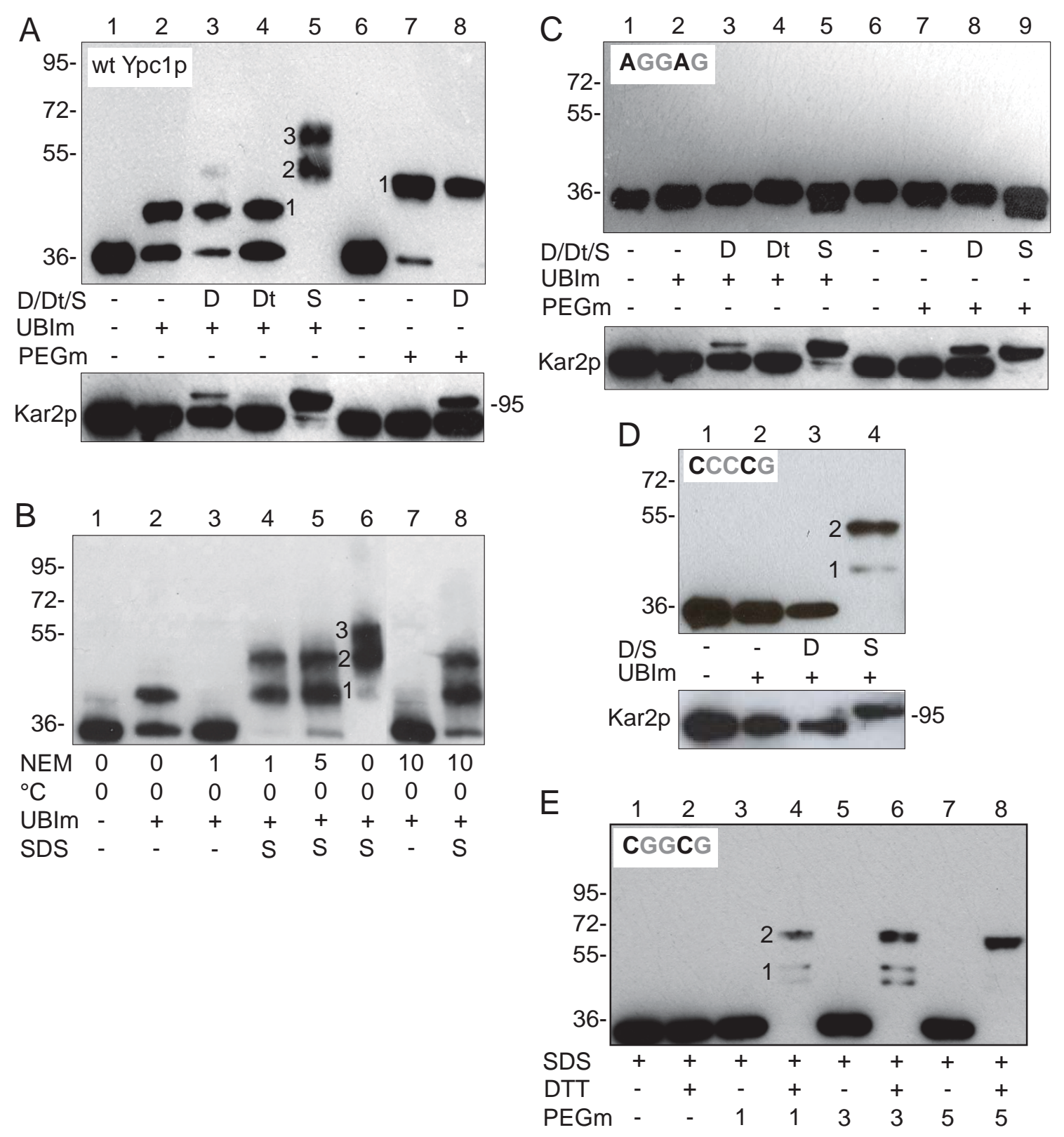

Fig. 4 

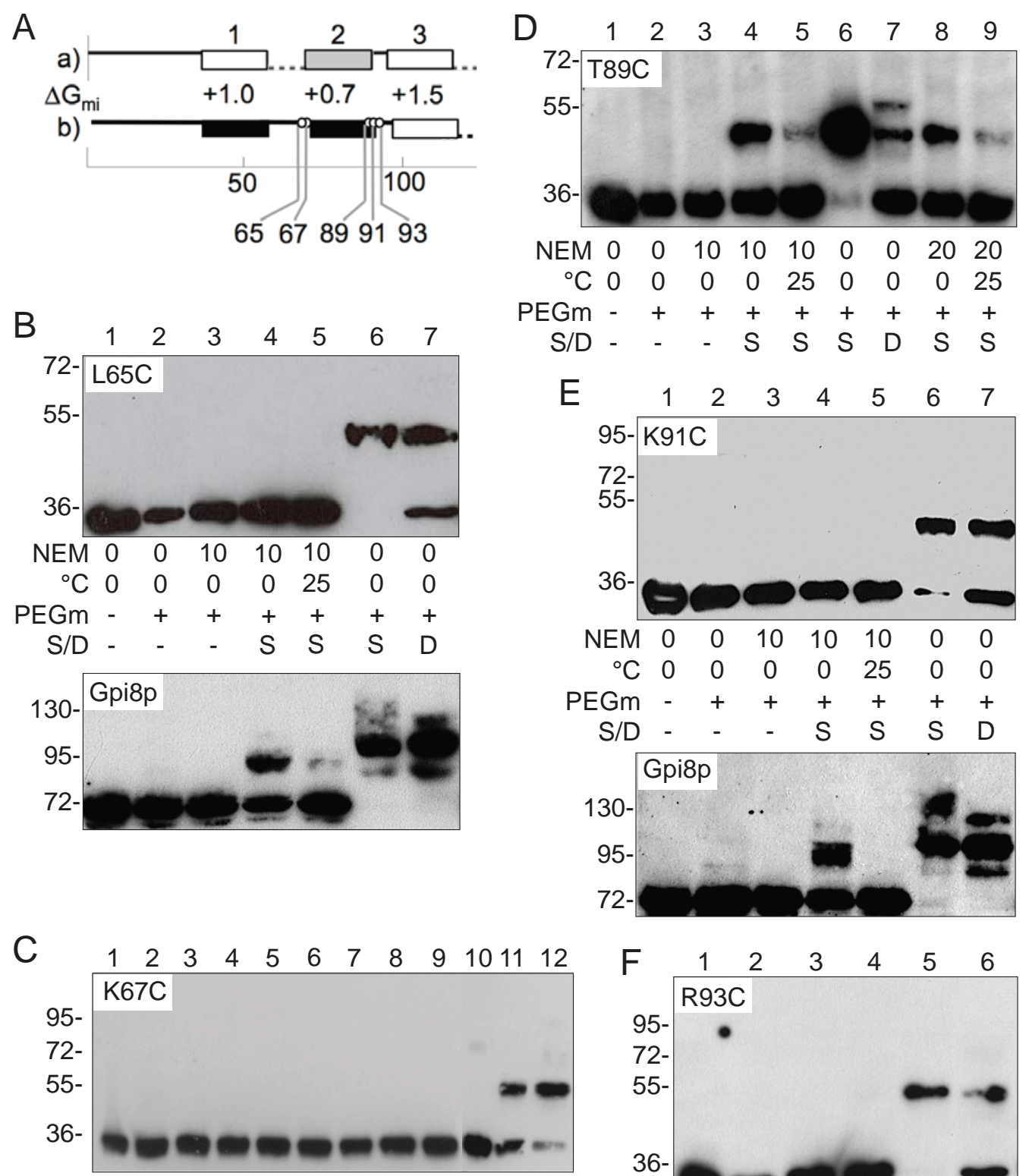

$\begin{array}{lllllllllllll}\text { NEM } & 0 & 0 & 0.3 & 1 & 5 & 10 & 0.3 & 1 & 5 & 10 & 0 & 0\end{array}$

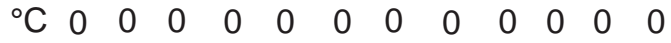
PEGm - + + + + + + + + + +
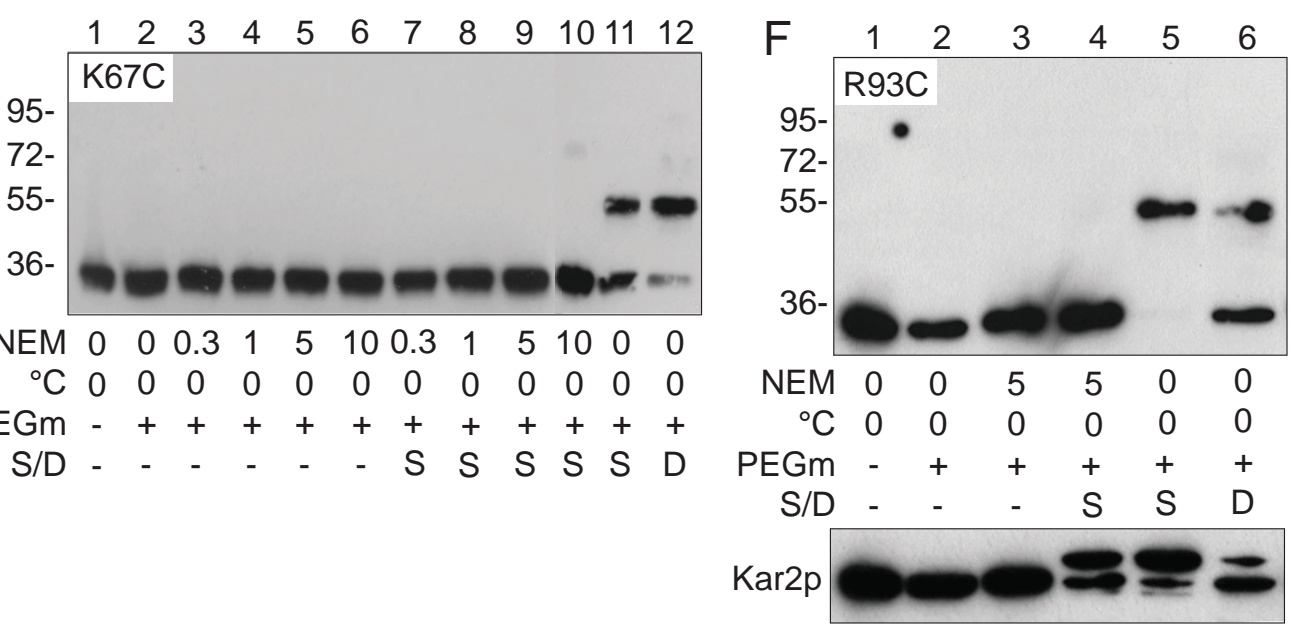

Fig. 5 

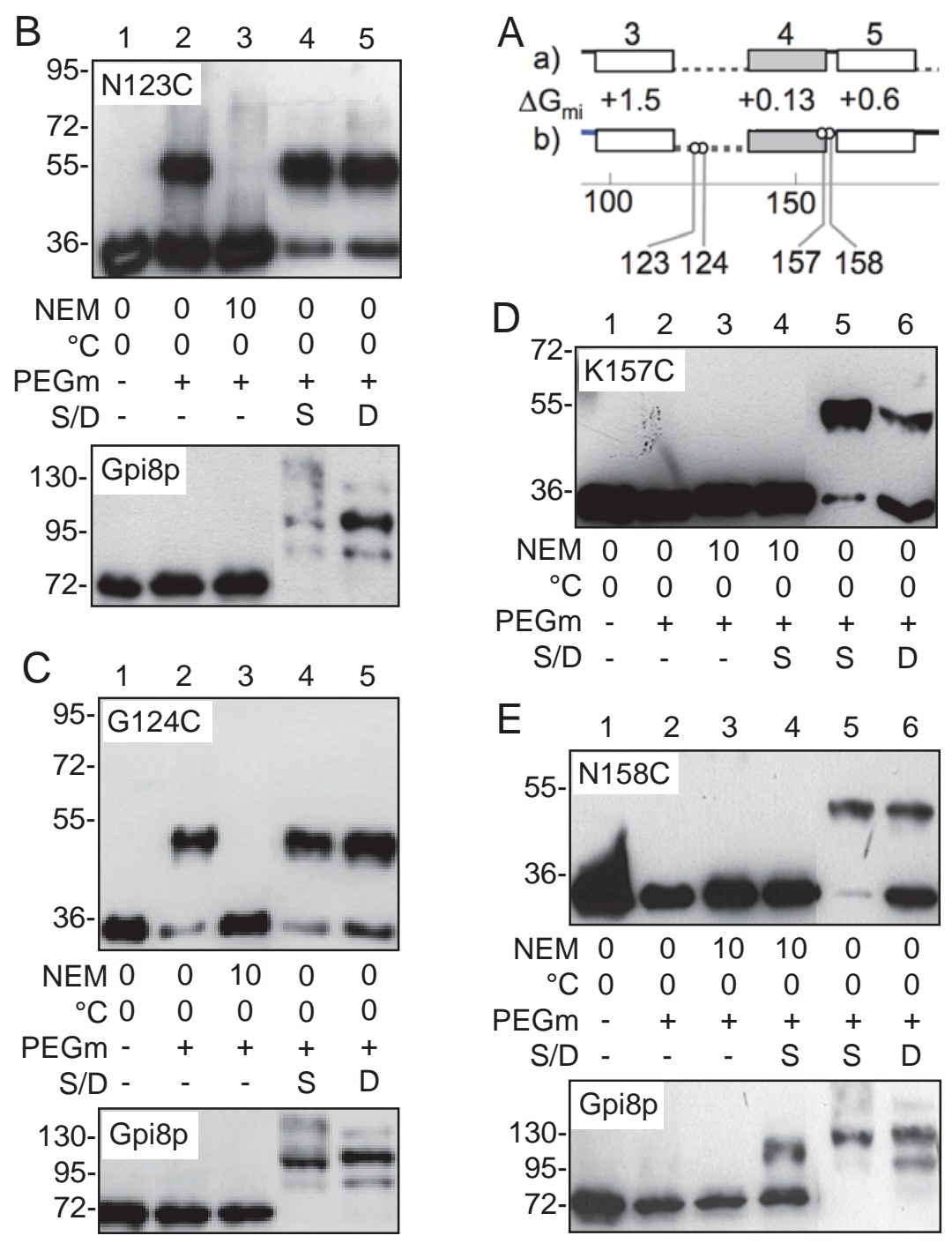

Fig. 6 
A

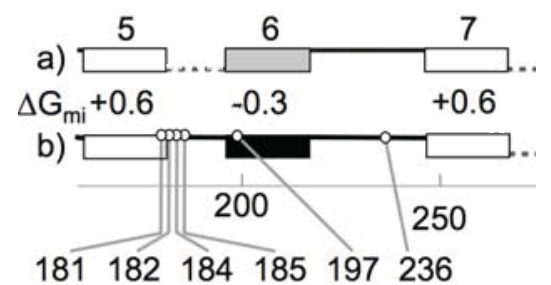

B

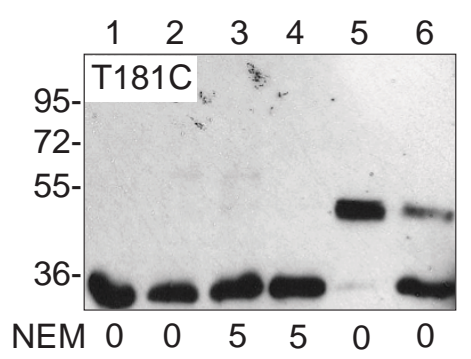

${ }^{\circ} \mathrm{C} \quad 0 \quad 0 \quad 0 \quad 0 \quad 0000$

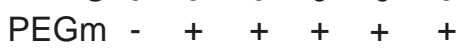

$S / D$ - $-S$ S D

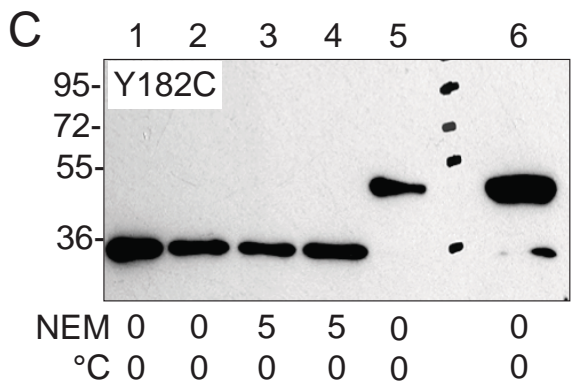

PEGm - + + + + +

S/D - - - S S D

$\begin{array}{llllllll}\mathrm{D} & 1 & 2 & 3 & 4 & 5 & 6 & 7\end{array}$

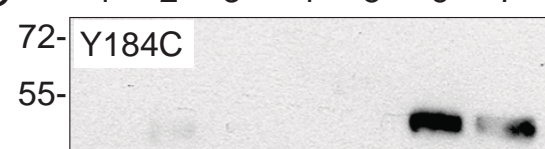

36-

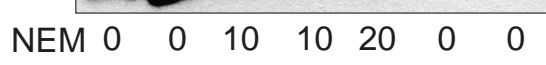

${ }^{\circ} \mathrm{C} \quad 0 \quad 0 \quad 000000$

PEGm - + + + + + +

S/D - - - S S S D

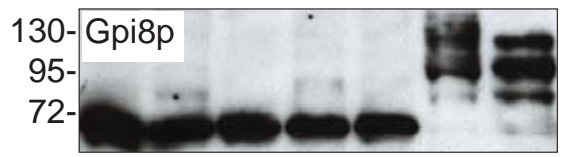

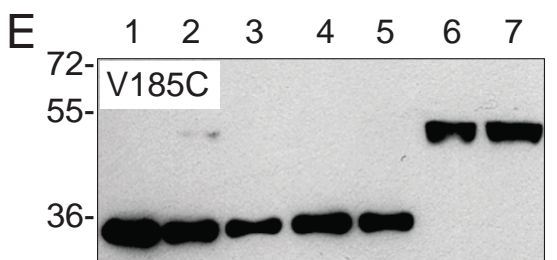

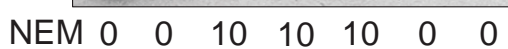

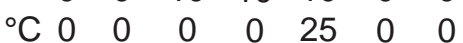

PEGm - + + + + + +

$S / D$ - $-S$ S $S$ D
F

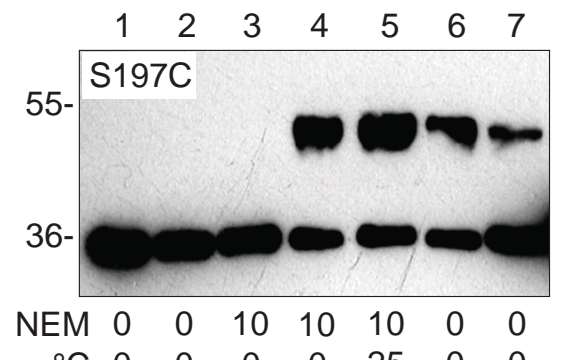

${ }^{\circ} \mathrm{C} \quad 0 \quad 0 \quad 000000$

PEGm - + + + + + +

$S / D$ - $-S$ S S D

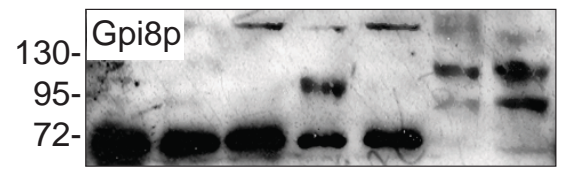

G
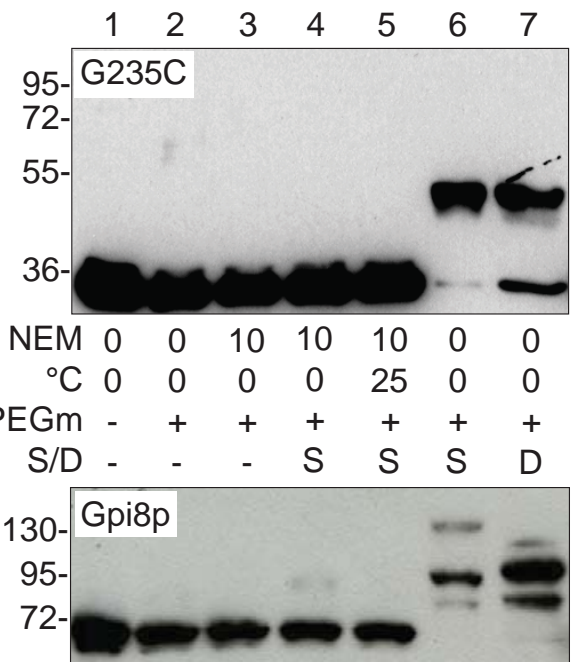

$\mathrm{H}$

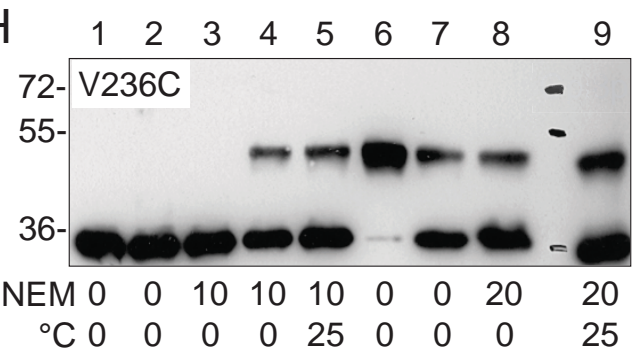

PEGm - + + + + + + + +

$S / D$ - $-S$ S S D S S

130- Gpi8p

$72-$

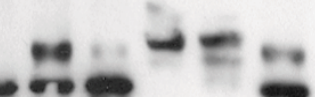

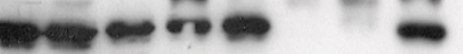

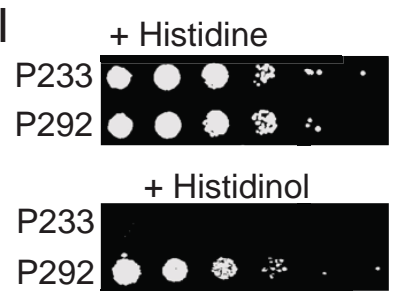

P233 P292

EndoH

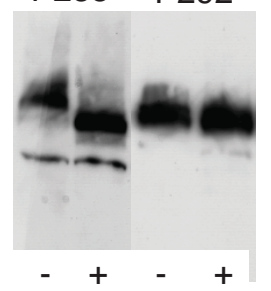

Fig. 7 


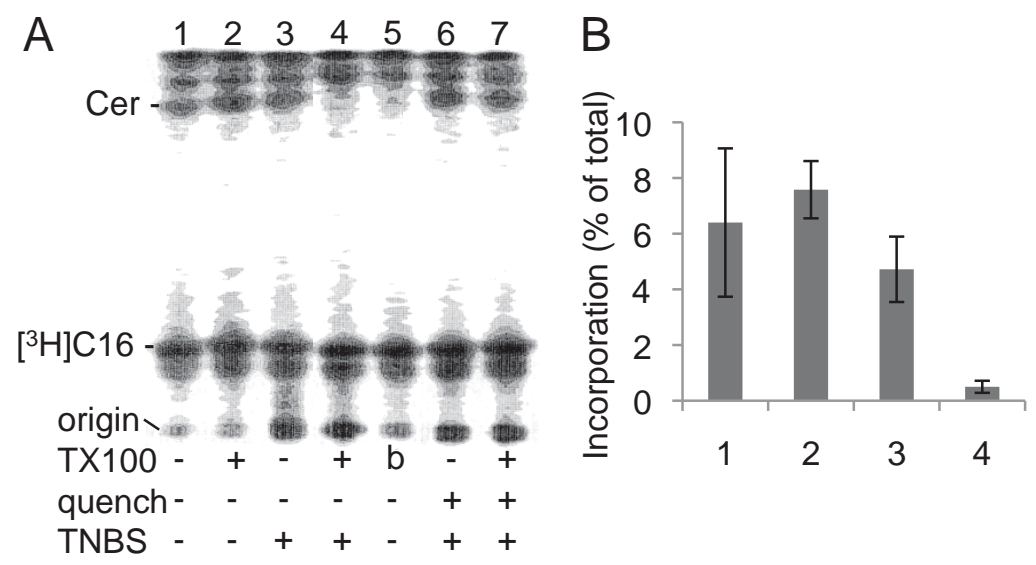

Fig. 8 


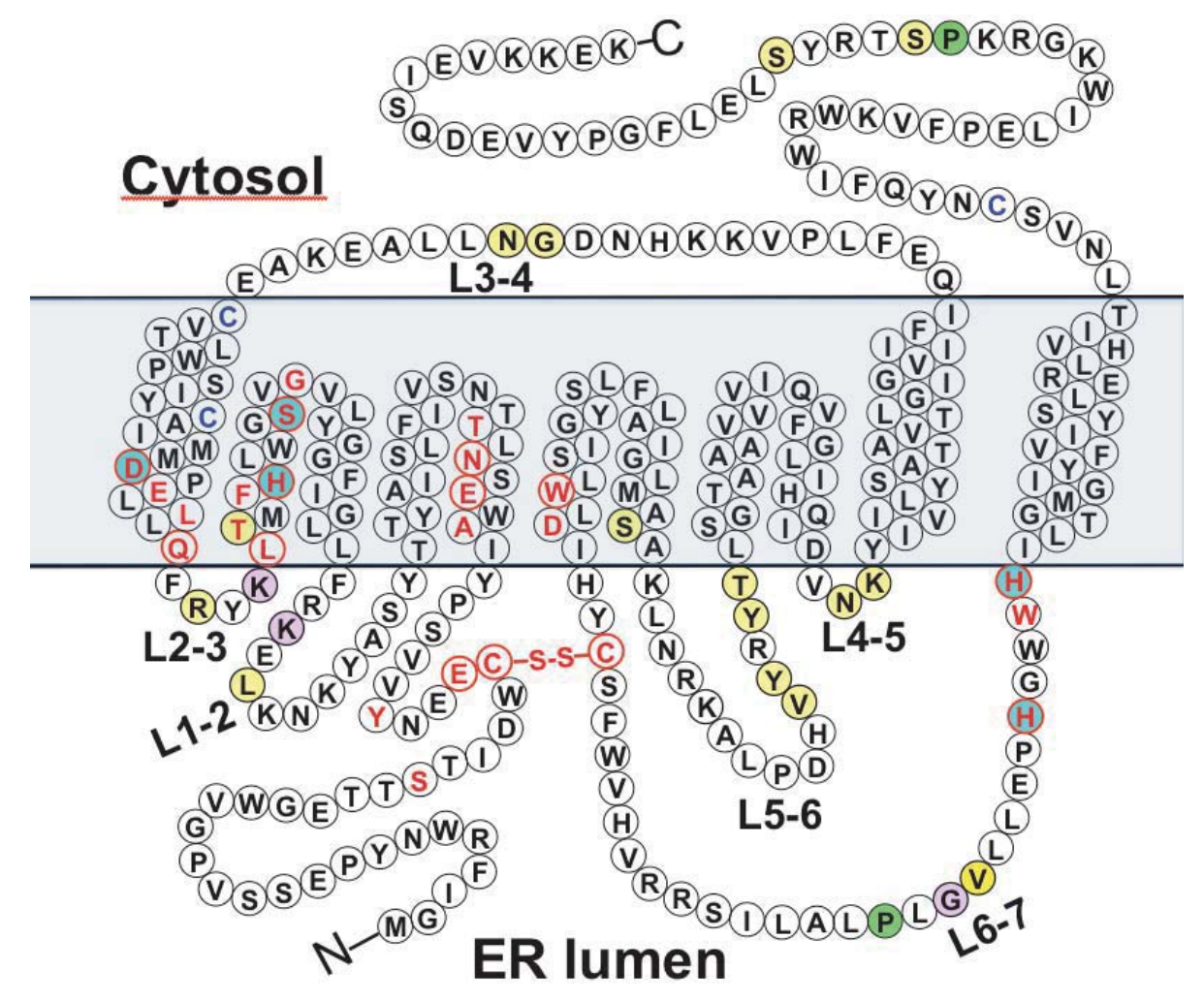

Fig. 9 